MILITARY TECHN. CAL COLLEGE CAIRO - EGYPT

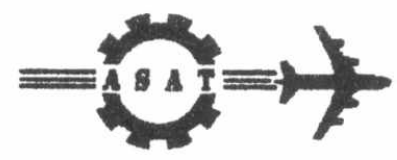

$7^{\text {th }}$ INTERNATIONAL CONF. ON AEROSPACE SCIENCES \& AVIATION TECHNOLOGY

\title{
AEROSPACE MATERIAL DAMAGE CHARACTERIZATION AND LIFE PREDICTIONS
}

\author{
B. M. RABEEH* W. O. SOBOYEJO ${ }^{\star *}$, S. I. ROKHLIN"**
}

\begin{abstract}
There is currently a concerted global effort to produce hypersonic vehicles. Structural materials in such vehicles must able to withstand high temperatures and retain a high stiffness, while carrying significant stresses. Titanium 15-3 metal matrix composites reinforced with SiC (SCS-6) fibers are being investigated to see if they satisfy the requirements for applications in such hypersonic vehicles. However, there is a limited understanding of structural failure modes in such composites. Fatigue damage mechanisms were identified in metal matrix composites via destructive and nondestructive testing (acoustic emission technique). Based on experimental evidence a micromechanical modeling approache was developped for the prediction of fatigue life in such composite materials. The model involves the use of crack-tip shielding concepts in the assessments of crack bridging phenomena during fatigue crack growth. In addition, an acoustic emission modeling was developped utilizing micromechanical modeling and fracture mechanics concepts. Fatigue life predictions were obtained and compared with the actual/measured fatigue lives. The current approach of non-destructive characterization to damage history and life prediction will lead to a new maintenance philosophy under realistic service conditions. Characterization as well as the location of fatigue damage under real service conditions will allow the airframe to utilize condition-based maintenance instead of programmed-depot maintenance.
\end{abstract}

* Department of Automotive Engineering, Military Technical College, Cairo, Egypt.

** Department of Materials Science and Engineering, The Ohio State University, Columbus, Ohio, USA.

** Department of Industrial Welding and System Engineering, The Ohio State University, Columbus, Ohio, USA. 


\section{INTRODUCTION}

Destructive Characterization of material behavior plays a vital development of materials and in understanding behavior of new materials and materials currently in service. In addition, new materials will require a better understanding of material response over the life time of the system. The bulk damage modes andidamage chatacterization will be studied using acoustic emission (non-destructive chatacterization). This include being able to correlate the changes in the material response that result in changes in the overall structure. Fatigue damage mechanisms were identified in Ti-15-3 monolithic matrix and Ti-15-3 metal matrix composites (MMC) reinforced with SiC (SCS-6) fibers separetly. A servo-hydraulic testing machine was conducted to the mechanical testing simaltanuously with acoustic emission test unit. Scanning electron microscopy observations were used to correlate acoustic emission analysis.

The damage and failure have tended to be addressed in terms of purly micromechanical changes attributed to mechanicl phenomena. Micromechanical modeling was developped based on understanding damalge meckanisms in both monolithic matrix and metal matrix composite separetly. The complex sequence of interfacial, matrix and fiber cracking is difficult to model exactly: Besides; the interactions between the multiple configurations of crack is also difficult to model within a conventional fracture mechanics framework. However, a simple idealization of the damage sequence can be obtained by recognizing that the damage mechanisms generally initiate from the outer surfaces (in monolithic matrix) and from the outer plies (in metal matrix composites) prior to subsequent propagation to the center or inner plies.

Early detection of acoustic emission is critical for the prevention of catastrophic failure, especially for metallic structures subjected to cyclic loading. Much effort has since been directed towards characterizing of the behavior of specimens linder loads [1-14]. This type of analysis has in tern lead to correlations between acoustic emissions and parameters characterizing the state of stress at crack tip and plastic zone ahead of the crack. Acoustic emission modeling was built based on the actual damage observations and the micromechanical modeling. It utilizes a combind contribution from the two mechanisms of microplasticity (dislocation "movements) within plastic zone, and crack tip extensions. The results of acoustic emission modeling were compared with that obtained from continuous acoustic emission and mechanical testing. The results of acoustic emission modeling suggests its benificial importance 
of damage monotoring and life predictions in the current aircracft applications. Hidden and inaccessable damage that do not produce vissual indications can now be detected and easily located within structural aerospece frame before it grows and produces vissually observable indications, and detectable lengths without the need to disassembly. Other environmental effects will now be taken into consideration beside fatigue such as corrosion, corrosion fatigue, and others.

\section{MICROMECHANICAL MODELING AND LIFE PREDICTIONS}

\section{Fatigue Life Prediction of Monolithic $\mathrm{Ti}(15-3)$ Matrix.}

In this section, we will demonstrate how fatigue lives can be predicted using mechanistically-based micromechanical models. The predictions obtained from the models will be compared with experimental data obtained from matrix material annealed at $815^{\circ} \mathrm{C}$ for 100 hours prior to air cooling. Experimental results [1] showed that matrix cracking initiated in the monolithic matrix at approx. half of the total fatigue life $(0.5 \mathrm{Nf})$. Consistent with the experimental observations, matrix cracking is assumed to initiate from the outer surface of the sample. The matrix fatigue cracks then propagate from the sample edge to the center where the cracks from two sides of the sample coalesce, i.e. the sample fails [1]. A schematic illustration of fatigue damage behavior is presented in Fig. 1.

Let us consider matrix crack initiation from the outer surface of the sample. The average crack length of the initiated crack (ao) was found to be $133 \mu \mathrm{m}$ for $815^{\circ} \mathrm{C}$ annealed sample [1]. The fatigue life of $\mathrm{Ti}$ (15-3) monolithic matrix can be predicted using the following Paris law expression which was obtained by curvefitting of data presented in Reference [2]:

$$
\frac{\mathrm{da}}{\mathrm{dN}}=2.931 \times 10^{-10}\left(\Delta \mathrm{K}_{\text {tip }}\right)^{3.381} \mathrm{~m} / \text { cycle }
$$

Separating variables:

$\mathrm{da}=2.931 \times 10^{-10}\left(\Delta \mathrm{K}_{\text {tip }}\right)^{3.381} \mathrm{dN}$

Integrating Equation 2 between appropriate limits, the fatigue life can now be predicted as: 
$\underset{N_{0}}{N_{f}} d \int_{d_{0}}^{\mathrm{a}_{f}} \frac{1}{2.931 \times 10^{-10}\left(\Delta \mathrm{K}_{\text {tip }}\right)^{3.381}} \mathrm{da}$

where $\Delta k$ is the effective stress intensity factor $(\mathrm{MPa} \sqrt{\mathrm{m}})$ and $N_{0}$ is the number of cycles required for crack initiation (obtained from acoustic emission experiments and scanning electron microscopy examination to be approx. half of total fatigue life $N_{f}$ ). The $a_{0}$ and $a_{f}$ are the corresponding crack lengths for the damage initiation and sample failure (at 0.5 of sample thickness) respectively. These can be related to the crack tip stress intensity factor values for monotonic loading, $K_{\text {tip }}$, using standard fracture mechanics formulae [3]. The stress intensity factor, $\Delta K_{\text {tip }}$, for an edge crack is given by the following empirical formula [3]:

$$
K_{\text {tip }}(a)=\sigma \sqrt{\pi a} \frac{1.122-0) .561\left(\frac{a}{i_{\max }}\right)-0.015\left(\frac{a}{i_{\max }}\right)^{2}+0.091\left(\frac{a}{i \max }\right)^{3}}{{\sqrt{1-i \frac{d}{c_{\max }}}}^{3}}
$$

where, $a$; is the crack length and $C_{\max }$ is the maximum crack length (half of the sample thickness). The above equations for stress intensity factor, $K_{\text {tip }}$, can be used to estimate the crack driving force where is no crack-tip shieiding. For cases when crack-tip shielding occurs via crack bridging under cyclic loading, the analysis of McMeeking and Evans [4] can be used to estimate $\Delta K_{\text {tip. }}$ A stress ratio, $R=$ $\mathrm{K}_{\min } / \mathrm{K}_{\max }=0$, is assumed in this analysis. Nevertheless, the analysis of McMeeking and Evaris [4] was used in the current study in which the stress ratio, $R$, was 0.1 . The near tip intensity factor for cyclic loading [4] is given by:

$\Delta \mathrm{K}_{\text {tip }}(\mathrm{a})=2 \mathrm{~K}_{\text {tip }}(\mathrm{a})$

where, $K_{\text {tip }}$ represents the near-tip stress intensity factor evaluated for the appropriate crack configuration subjected to monotonic loading (Fig. 1). Substituting Equation 5 into Equation 3, it is relatively easy to compute $N_{f}$ by numerical integration when all the variables listed in Equation 3 are known. From previous experimental work [1], the initial crack lengths, $a_{0}$, were measured to be in the range given by $\left(122 \mu \mathrm{m} \leq a_{0} \leq\right.$ $144 \mu \mathrm{m}$ ). The measured range of af values (to be approx. $1150 \mu \mathrm{m}$ ) will be used 


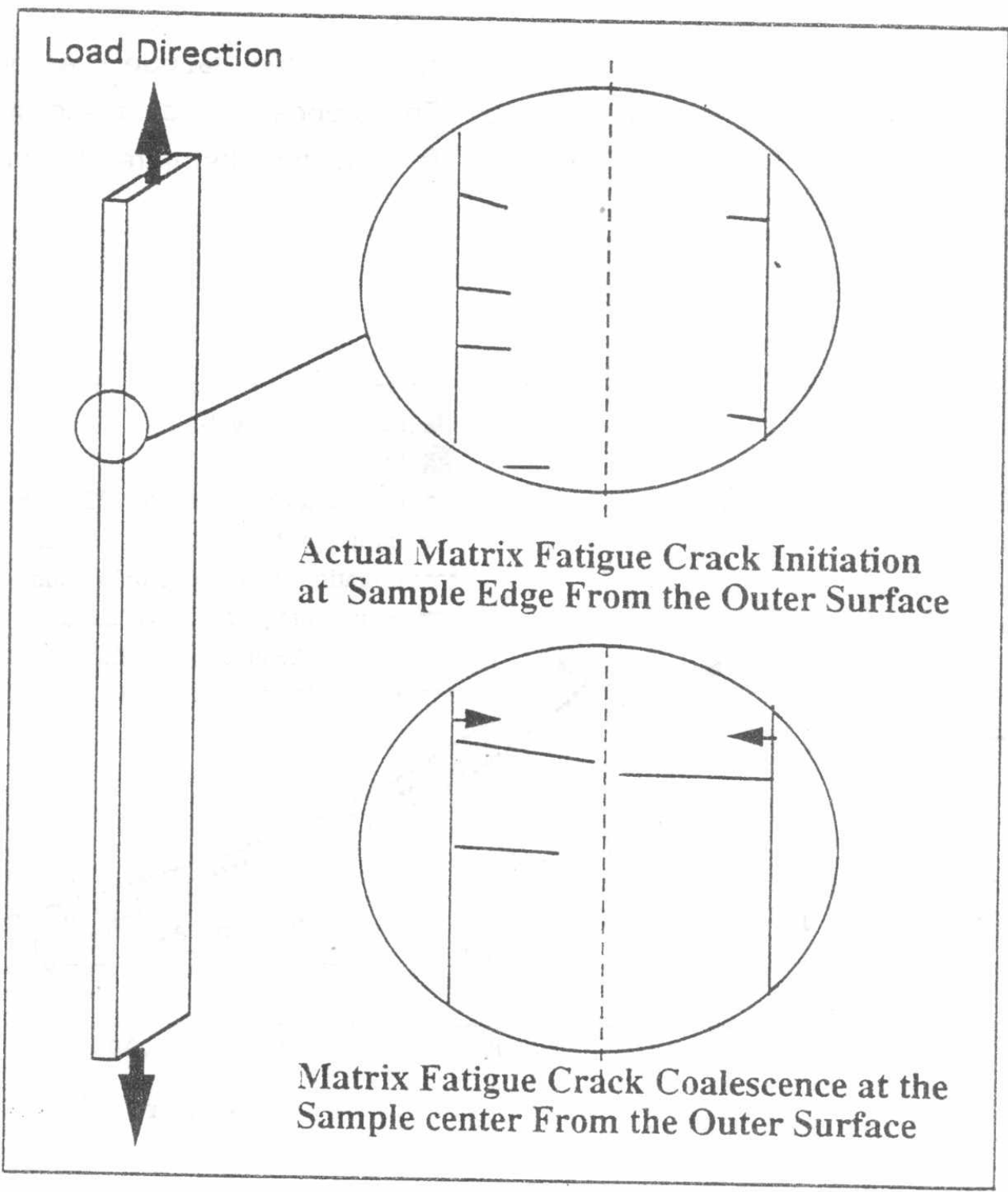

Figure I: Schematic Illustration of Idealized Fatigue Crack Initiation and Coalescence at Monolithic Matrix $\mathrm{Ti}(15-3)$ Materiais Annealed at $815^{\circ} \mathrm{C} / 100 \mathrm{H} / \mathrm{AC}$ Used in Modeling
of Damage Under cyclic Loading.

in the prediction of fatigue propagation life, $\mathrm{Np}$. Now, since the total fatigue life, $\mathrm{Nf}$, is the sum of cycles prior to crack initiation $\mathrm{Ni}$ (found by acoustic emission experiment to be approx. half of $\mathrm{N}_{f}$ ) and number of cycles for crack propagation $\mathrm{N}_{p}$, the total fatigue life, $\mathrm{N}_{f}$, can be estimated from $2 \mathrm{~Np}$. The initiation fatigue life can also be approximated since $\mathrm{Ni} \sim \mathrm{N}$. The predicted initiation and total fatigue lives are compared with the measured lives obtained from the specimens annealed at $815^{\circ} \mathrm{C} / 100 \mathrm{H} / \mathrm{AC}$. The predicted fatigue lives are presented in Fig. 2 for the complete range of different crack lengths, $a_{0}$. Excellent agreement is observed between the 
experime, ntal results (actual fatigue lives) and the predicted fatigue lives obtained from t'ne idealized fracture mechanics model. The proposed mechanistically-based fract'ure mechanics approach to Ti-15-3 matrix life prediction therefore appears to be ve.iid.

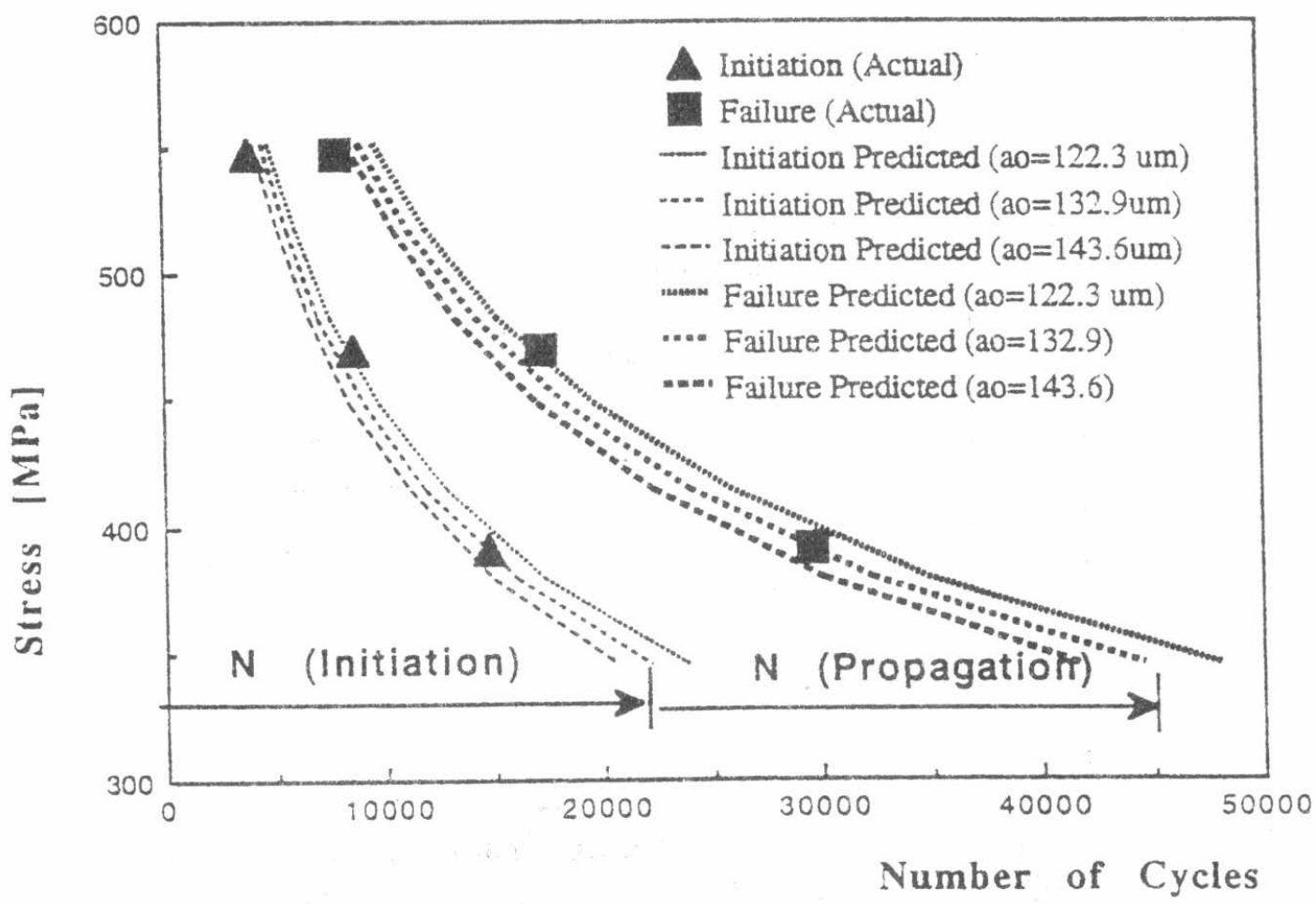

Figure 2: Comparison of Predicted S-: V Curves (Theoretical) and the Experimentally Measured S-N Curve For Monolithic Ti $(15-3)$ Matrix Annealed at $815^{\circ} \mathrm{C} / 100 \mathrm{H} / \mathrm{AC}$.

\section{Fatigue Life Prediction of $[0 / 90]_{2 S}$ Ti MMC}

A simple idealization of the damage sequence [1] can be obtained by recognizing that the damage generally initiates in the outer plies prior to subsequent propagation into the inner plies. The initial debonding in the outer zero degree plies [1] can be idealized as a single eccentrically-located center crack with initial length equal to the fiber diameter. The initial crack is assumed to extend without interacting significantly with surrounding cracks. It is assumed to be equal to the fiber diameter (Fig. 3a). Next it is also assumed to propagate as a through-thickness center crack until it 
extends completely through the first ply (stage 1). The crack is then recharacterized as an edge crack (Fig. 3b) and allowed to extend as an unbridged crack until it reaches the first raw of $0^{\circ}$ fibers in the second ply (stage 2). The edge crack is then assumed to be bridged by the fibers in the second ply until it reaches. the fourth ply (Fig. 3c). The configuration shown in Fig. $3 \mathrm{c}$ is then maintained until the crack reaches the boundary between the fourth and fifth plies (Fig. 3d). At this stage, crack coalescence occurs when the propagating cracks from both sides of the sample meet (stage 3). Note that the final condition for catastrophic failure could also be assumed to occur when $\mathrm{K}_{\text {tip }}$ reaches the matrix fracture toughness, $\mathrm{K}_{\mathrm{IC}}$. However, the errors introduced by the assumptions in stage 3 are relatively small due to the fast growth rates in this regime.

Before presenting the results of the analysis, it is important to discuss the simplifying assumptions that were made in the idealized fracture mechanics model. First only fibers in the $0^{\circ}$ plies are assumed to bridge the crack. Also the possible effects of the variations in opening displacement on fiber fracture and bridging characteristics have been neglected, i.e., the bridge lengths were equal to the distance between the crack-tip and the last first row of bridging fibers. It was also assumed that the fibers in the $90^{\circ}$ plies did not have a significant effect on the mode 1 crack path, although it is quite clear that such fibers may promote crack deflection (by tilting and twisting) since the modulus of the SCS-6 fiber is higher than that of the matrix [5-6]. Finally, the possible effects of residual stress [7-8] were neglected in the analysis, and the calculations were carried out for the as-received composite, since the matrix and the fiber mechanical property data were available for material in this condition.

The fatigue lives of SCS-6/Ti (15-3) composite can now be predicted by integration of Paris law expression (Equation 3), as discussed in the previous section. Considering the first stage of fatigue damage as center crack in the outer ply (Fig. 3a), the stress intensity factor $\Delta K_{\text {eff }}$ is approximately equal to $2 K_{\text {tip }}$ (equation 5). The near tip stress intensity factor, $K_{\text {tip }}$, for monotonic loading of sample with center crack is given [9] as:

$K_{\text {tip }}(a)=\sigma \sqrt{\pi a} \frac{1-0.5\left(\frac{a}{c_{\max }}\right)+0.326\left(\frac{a}{c_{\max }}\right)}{\sqrt{1-\left(\frac{a}{c_{\max }}\right)}}$ 


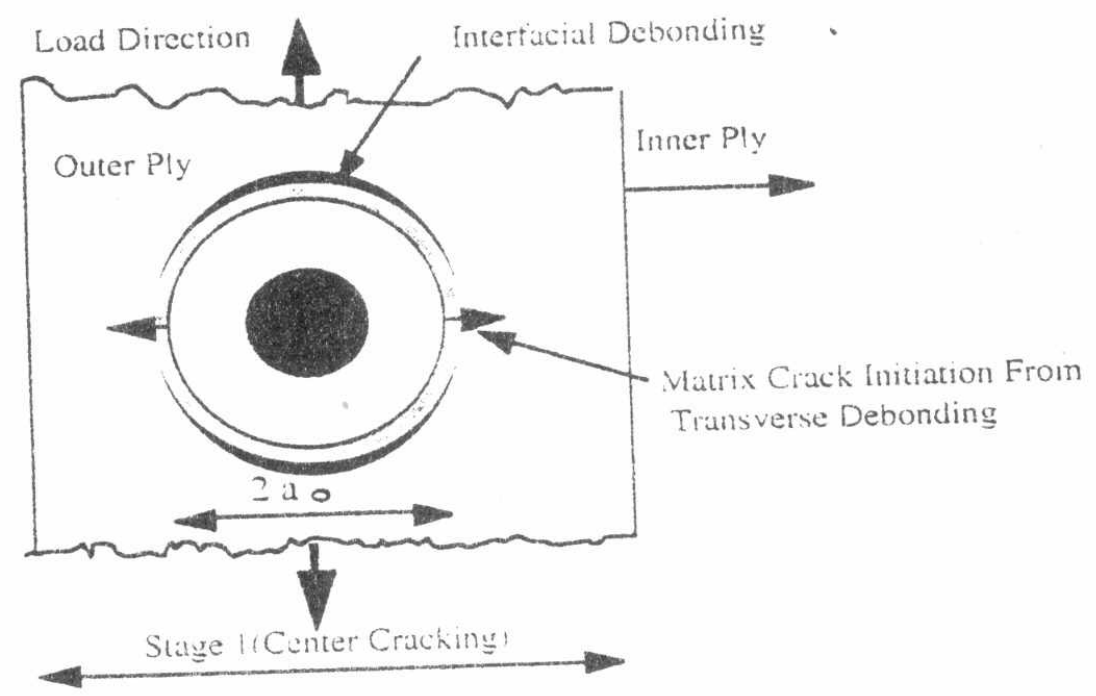

(a)

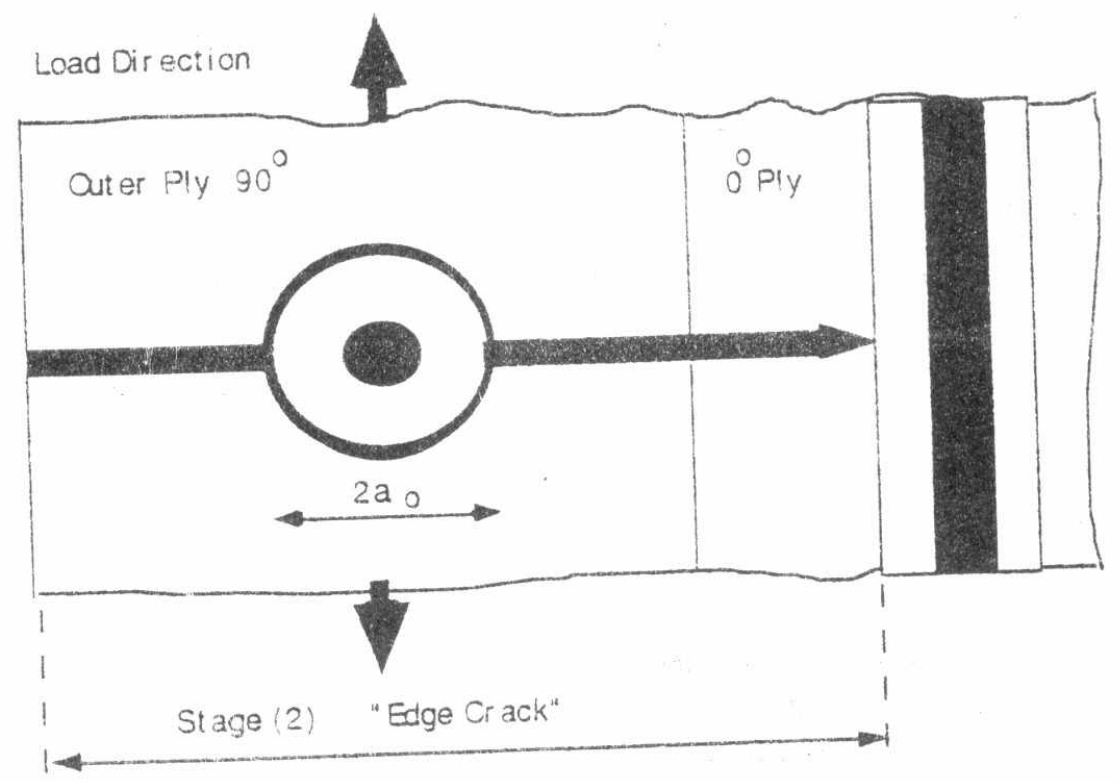

(b)

Figure 3: Schematic lllustration of Idealized Crack Growth and Crack Bridging Configurations Used in Modeling of Damage Under cyclic Loading: (a) Center Crack Initiation From Outer (900) Plies (Stage 1) and (b) Edge Crack Propagation From Outer Plies. 


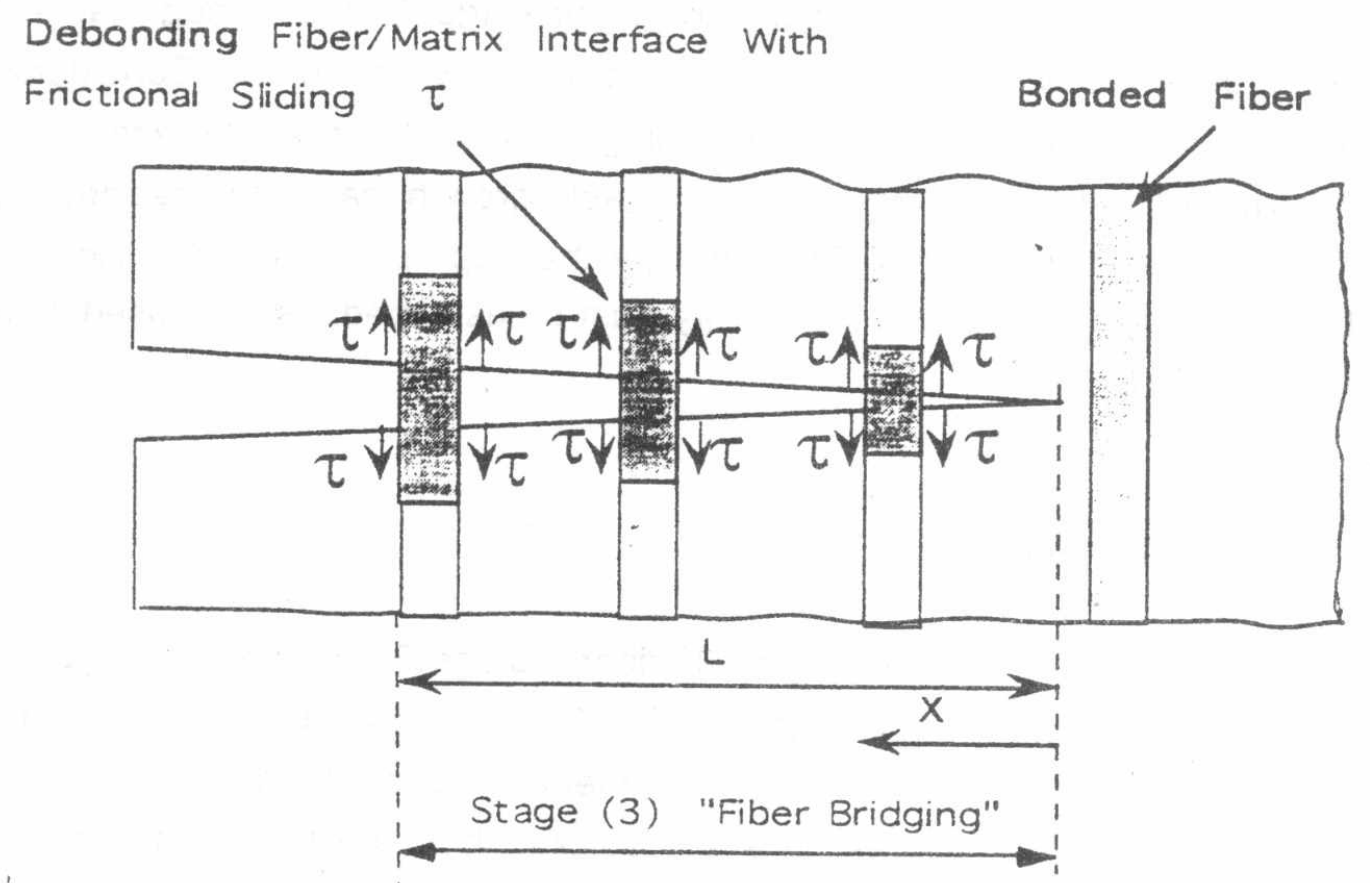

(c)

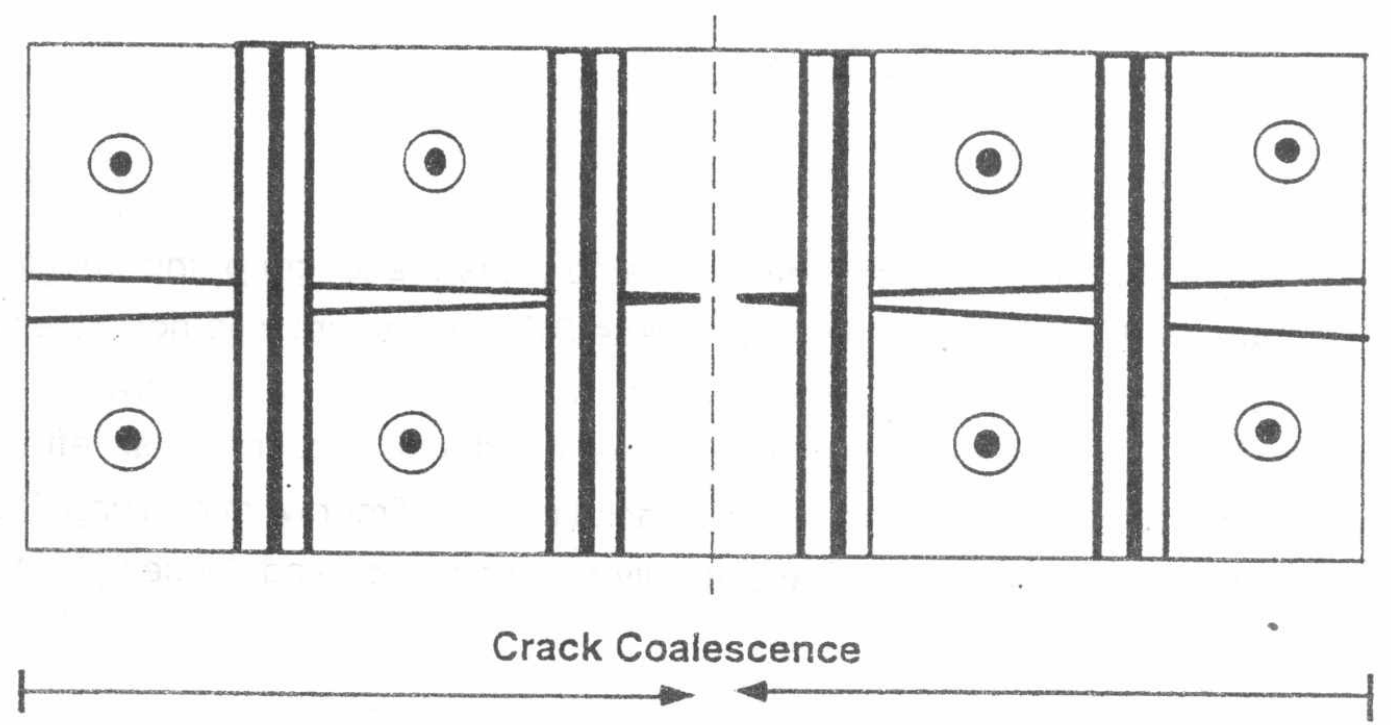

(d)

Figure 3: Schematic Illustration of Idealized Crack Growth and Crack Bridging Configurations Used in Modeling of Damage Under cyclic Loading: (c) Edge Cruck Propagation Into Inner Plies, and (d) Crack Coalescence at the Center of Inner Plies. 
where, $a$; is the crack length and $C_{\max }$ is the maximum crack length (haif of the ply thickness). The stress intensity factor, $K_{\text {tip }}$, for stage 2 (double edge fatigue crack growth) is given by the same empirical formula (Equation 4). Considering the analysis of McMeeking and Evans [4]. Using the near tip intensity factors for cyclic loading for both stage 1 and stage 2, crack growth can now be predicted by substituting Equations 4-6 into Equation 3 at the appropriate stages. However, the shielding effects of crack bridging on $\Delta K_{\text {tip }}$ must be assumed, as discussed in the next section.

\section{Fiber Bridging Analysis}

The shielding due to crack bridging by 00 fibers (stage 3) can be modeled using the analysis by Hutchinson and Jensen [12]. A simplified case of constant friction (bridging traction) was used in the assessment of crack bridging. This yields the following expression for the stress intensity factor at the crack tip, $k_{t i p}(a)$ is given by:

$$
K_{\text {tip }}(a)=K_{\text {app }}-\sqrt{\frac{2}{\pi}} \int_{0}^{L} V_{f} C\left(\frac{K_{t i p} \tau}{D}\right)^{\frac{1}{2}} X^{\frac{1}{4}} d X
$$

where $K_{a p o}$ is the applied stress intensity factor, $L$ is the length of the bridging zone which is equated to the crack length at stage 3 (fiber fracture was not observed until sample failure), $V_{f}$ is the volume fraction of fiber, $C$ is the constraint/hardening parameter, $D$ is the fiber diameter, $\tau$ is the interfacial friction stress after fatigue damage, and $X$ is the distance from the crack-tip to the first raw of bridging fibers (Fig. 3c). Equation 7 had been solved analytically by Hutchinson and Jensen [12] to give:

$$
K_{\text {tip }}(a)=\frac{1}{4}\left[\sqrt{b^{2}+4 c-\cdots b}\right]
$$

where $b$ is given by [12]:

$$
b=\frac{16}{3 \pi}\left[\frac{2 \cdot \overline{2}\left(1-v^{2}\right) E^{2} v_{f}}{\left(b_{2}+b_{3}\right)\left(1-v^{2}\right) A E_{m}}\right]^{\frac{1}{2}} \times\left(\frac{L}{D}\right)^{\frac{3}{4}}[\tau \cdot \overline{\pi D}]^{\frac{1}{2}}
$$


and $\mathrm{C}=\mathrm{K}_{\mathrm{app}}$

where, $A$ is the orthotropy factor, $v$ is the poison ratio of the matrix, $b_{2}$ and $b_{3}$ are fiber constraint parameters that can be obtained from Refs. [4,10], $E^{\prime}$ is the composite Young's modulus estimated from constant strain-rule-of-mixtures using matrix modulus $\left(E_{m}\right)$ and fiber modulus $\left(E_{f}\right)$ values. $E^{\prime}$ is thus given by;

$E^{\prime}=V_{m} E_{m}+V_{f} E_{f}$

where, $V_{m}$ and $V_{f}$ are volume fraction of matrix and fiber respectively.

The frictional stresses on the individual sliding interfaces are assumed to be equal to the mean value of individual frictional stresses. The $[0 / 90]_{2 S}$ fiber composite is also assumed to be orthotropic material [11]. The effects of interface friction stress, $\tau$, and the fiber stresses within the bridging zone are described by equation 8 . The total fatigue life is obtained when matrix crack coalescence occurs at the center of the inner plies (sample center) and composite fails (Fig. 3d).

The fatigue life of the as-received composite can now be predicted by integration of Paris formula (Equation 1). The fatigue lives of the as-fabricated composite specimens are thus estimated by solving the Equation 3 after substitution of appropriate material property data. Constant interfacial friction stresses, $\tau$, of 35,50 , 200, 250 and $300 \mathrm{MPa}$ were employed in the calculations. These values correspond to data reported in the literature $[12,13]$ for as-received $\mathrm{Ti}-15-3 / \mathrm{SiC}$ composite. Also our push out experiments yielded average interfacial shear stress, $\tau=265 \mathrm{MPa}$. The typical range of $\tau$ that was measured in the push-out experiments [1] was between 200 and $300 \mathrm{MPa}$.

The predicted fatigue lives (S-N curves) are presented in Fig. 4. The predicted S-N curves obtained using interfacial strength values between $200 \mathrm{MPa}$ and $250 \mathrm{MPa}$ are in close agreement with the S-N data obtained from the fatigue experiments performed on as-received material. This is close to interfacial strength, $\tau$, of fiber push-out tests which was about $265 \mathrm{MPa}$ (overall value for the outer and inner plies). Notched specimens have been shown to have lower interfacial shear strengths due to possible interfacial wear phenomena that can occur during fatigue 
damage [14]. The lower bound estimates of interfacial friction strength (35 MPa and $50 \mathrm{MPa}$ ) which account for the effects of fatigue damage of interface [14]. The predicted fatigue lives using $\tau=35$ and $50 \mathrm{MPa}$ are lower than the measured by fiber push-out test. However, similar interfacial friction strength degradation mechanisms have not been reported for smooth specimens deformed under cyclic loading. This suggests that the fatigue-induced degradation of interfacial strength may not be very significant in the un-notched specimen examined in this study. Differences between the degree of degradation of interfacial shear strength in smooth and notched specimens may be due to the higher crack opening displacements in notched specimens.

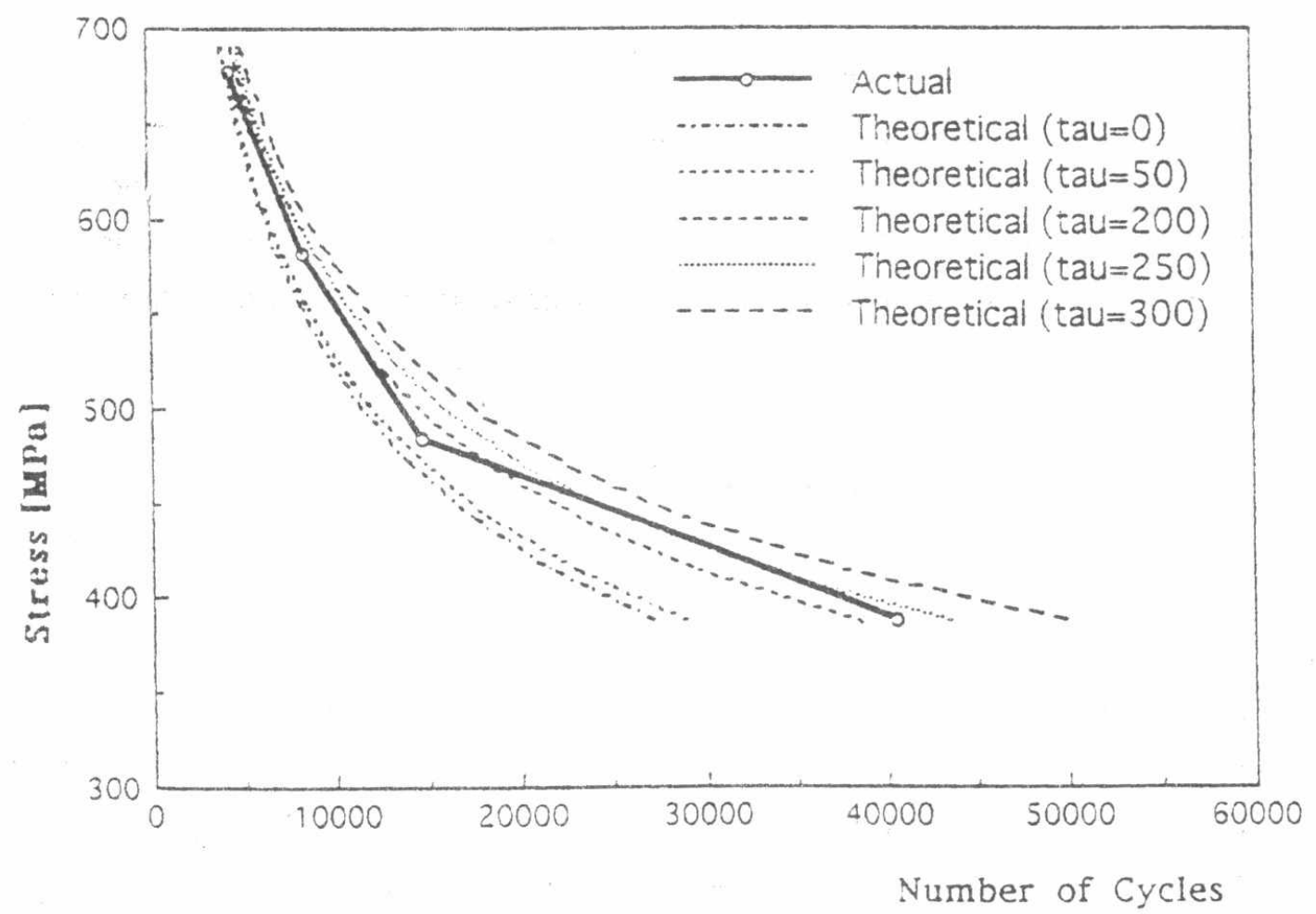

Figure +: Comparison of Predicted S-N Curves (Theoretical) at Different Interfacial Friction Stress $(\tau)$ and the Experimentally Measured S-N Curve For As-Received Ti (15-3)MMC.

The predicted S-N curves obtained for the composite with $\tau=200 \mathrm{MPa}$ are compared with the actual experimental data obtained from Ti (15-3) MMC and monolithic Ti (153) matrix in Fig. 5. The MMC has longer fatigue lives than the monolithic matrix at 
the same stress ranges. It is also clear from the above arguments that the longer fatigue lives in the composite are due to the beneficial effects of crack bridging.

The apparent accuracy of the proposed fracture mechanics model is somewhat surprising given the simplicity of its formulation. Nevertheless, the current results do show the potential for the development of accurate fatigue life prediction methodologies for structures and components fabricated from titanium matrix composites. Above all, however, the work illustrates the importance of simplified mechanics-based models that attempt to stimulate actual experimental observations of damage. This technique may be extended to the currently air force applications for damage history and life prediction.

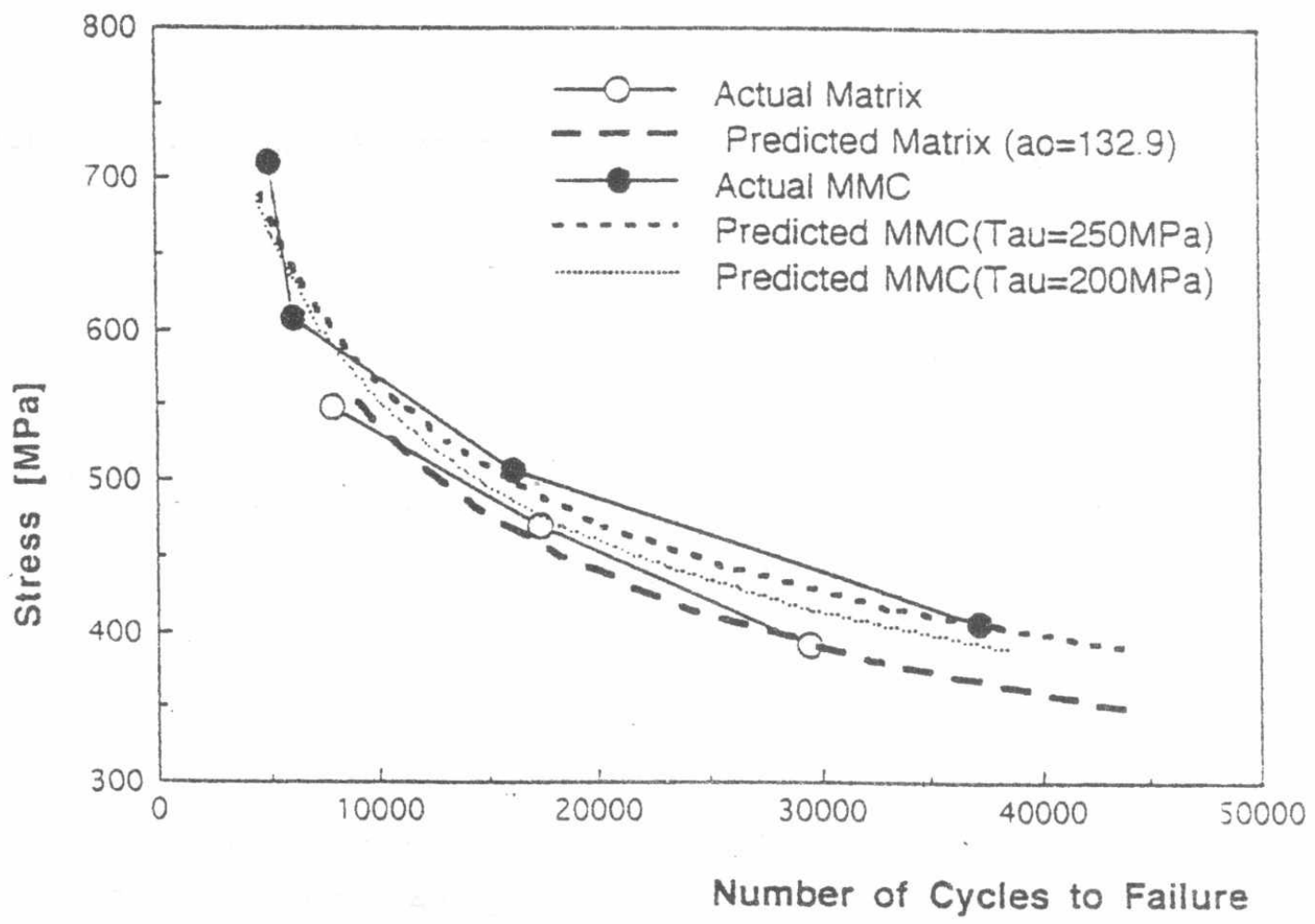

Figure 5: Comparison of Predicted S-N Curves (Theoretical) at Different Interfacial Friction Stress $(t)$ and the Experimentally Measured S-N Curve For $\mathrm{Ti}(15-3)$ Monolithic Matrix and MMC Annealed at $815^{\circ} \mathrm{C} / 50 \mathrm{H} / \mathrm{AC}$. 


\section{ACOUSTIC EMISSION MODELING AND LIFE PREDICTIONS.}

It has also been reported [15] that, depending on the ductility of the material and on the test conditions (the loading ratio $R=\sigma_{\min } / \sigma_{\max }$ ), a combination of plastic deformation and fracture mechanisms can be responsible for the overall acoustic emission signals/counts $[16,17]$. Hence the acoustic emission wave detected are either due to energy released during crack extension or due to the deformation and fracture within plastic zone. The combined contribution from these two mechanisms to the AE cumulative counts rate is the sum of; (1) The count rates due to plasticity (dislocation movements) within the plastic zones, $N_{p}^{\prime}[16]$ :

$$
v_{p}=C_{p} \Delta K^{m} \frac{\Delta K^{2}}{(1-R)^{2}}
$$

and (2). The count rate due to the creation of new surfaces during crack extension, $\mathrm{N}^{\prime} \mathrm{C},[17]$ :

$$
N_{C}^{\prime}=C_{S} \frac{\Delta K^{m}}{(1-R)^{m}}
$$

The overall count rate will, therefore, be:

$$
N_{\text {total }}^{\prime}=N_{p}^{\prime}+N_{c}^{\prime}
$$

where, constants. $C_{p}$ and $C_{s}$ are related to plastic yielding and crack extension respectively need to be determined from $A E$ measurements at appropriate stages of damage. Unlike precious work [15-18] the current study will demonstrate the general applicability of the above approach to the prediction of fatigue life in homogeneous (matrix) and heterogeneous (composite) materials. Numerical integration of Equations 12-14 can be used to estimate the fatigue life in terms of the number of counts to failure. The implications of such predictions will be discussed later. 


\section{Acoustic Emission Modeling of Fatigue Damage in Monolithic Matrix}

To predict the acoustic emission cumulative counts it is necessary to first obtain the constants $C_{p}$ and $C_{s}$ (used in Equations 12 and 13). The constants can be determined from the $A E$ data obtained from the fatigue crack initiation and propagation experiments [1]. These constants are related directly to material properties and sample geometry [17]. The measured value of constant $C p$ and $C_{s}$ (obtained from actual acoustic emission data) to be: $C p=9.671 \times 10^{-4}$ and Cs $=3.037 \times 10^{-3}$, can be substituted into Equations 12,13. The predicted acoustic emission data are compared to experimental data and presented in Fig. 6. The actual $A E$ results obtained at stress ranges of $0.5 \sigma_{U T}$ and $0.6 \sigma_{U T}$ are in very good agreement with the predicted counts. However, some differences are observed between the experimental data and the predictions obtained for stress range of $0.7 \sigma_{U T}$ (due to loose of transducer coupling)

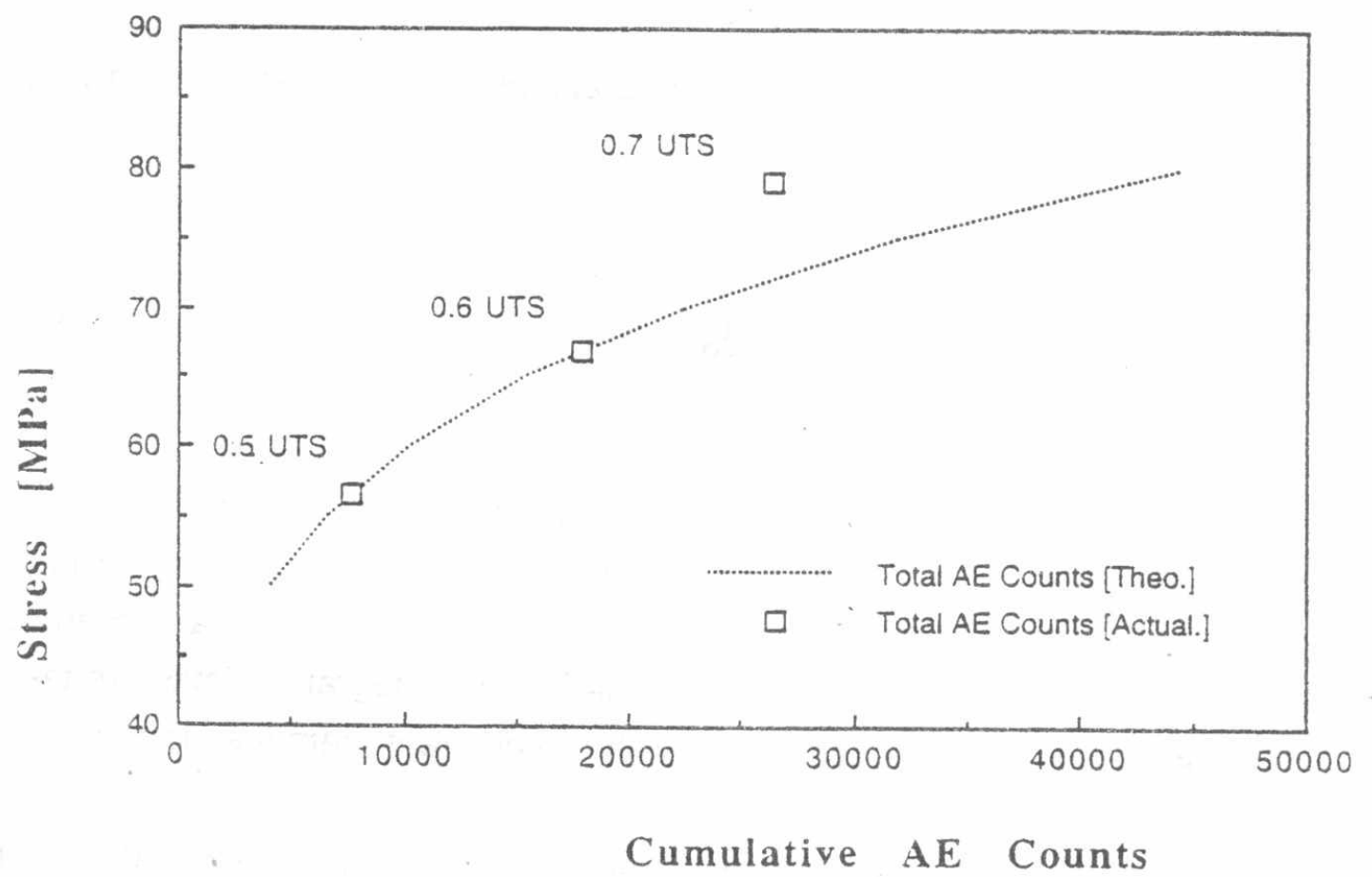

Figure 6: Comparison of Stress Versus the Predicted Number of Total AE Counts Obtained From Monolithic Matrix (Ti 15-3) Sample Annealed at 815$100 \mathrm{H}-\mathrm{AC}$. 


\section{Acoustic Emission Modeling of Damage Mechanism of $[0 / 90]_{2 S}$ Ti MMC}

The, acoustic emission model for the prediction of fatigue life in the as-received $\mathrm{Ti}$ (153) $M M C$ are presented in this section for stress ranges from $0.4 \sigma_{U T}$ to $0.8 \sigma_{U T}$. While the actual results are only availlable for stress ranges of $0.5 \sigma_{U T}$ and $0.7 \sigma_{U T}$. For each stage of damage mechanisms (mentioned in micromechanical modeling), the associated number of cycles was obtained. These number of cycles is considered as the integration limits for the prediction of the acoustic emission counts, at different damage stages. We consider bridging effects of fibers and crack length associated with each damage stage at micromechanical modeling. Acoustic emission modeling is now designed, as extension of micromechanical modeling, for fatigue life prediction. These acoustic emission counts classified into counts due to deformation and fracture within plastic zone is given as:

$$
v_{p i}=\int_{v_{i i}}^{v_{l i+1}} 1.332 \times 10^{-3}\left(\Delta K_{\text {eff }}\right)^{3.381} \frac{\Delta K^{2} \text { eff }}{(1-R)^{2}} d . V^{2}
$$

and acoustic emission counts due to crack tip extension (the creation of new surfaces) is also given as:

$$
V_{c i}=\int_{v_{f i}}^{v_{f i+1}} 4.1836 \times 10^{-3} \frac{\Delta K_{e f f}{ }^{3.381}}{(1-R)^{3.381}} d N
$$

The total number of the predicted acoustic emission counts is the sum of both the number of counts due to deformation and fracture within plastic zone (Equation 15) and the number of counts due to crack tip extension (Equation 16) at the end of each stage. The number of cycles $\mathrm{N}_{\mathrm{fi}}$ and $\mathrm{N}_{\mathrm{fi}+1}$ used as the integration limits are taken from the fatigue experiments limits associated with the different damage stages.

\section{Correlations Between Actual and Predicted Fatigue Life of AR Ti MMC}

The acoustic emission cumulative counts, obtained from experiments [1], are compared with the AE predictions in Fig. 7 . The predicted and experimental data are in excellent agreement. Based on the results [1] obtained from monolithic matrix, acoustic emission counts obtained from plastic deformation characterized by high 
frequency $(\geq 200 \mathrm{KHz})$ and low $\mathrm{AE}$ counts $(0-50$ counts), while crack extension was characterized by low frequency $(<200 \mathrm{KHz})$ and high $\mathrm{AE}$ counts ( $>50$ counts). However, although the close agreement between the measured and predicted counts is encouraging. It is important to note that further work is needed to confirm the general applicability of the above methods to other monolithic and composite material systems. Nevertheless, it is clear that the above methods may be used to quantify the deterioration of a structure/component during initiation/evolution. Quantitative numerical integration techniques may also used to predict remaining lives and inspection intervals when the required AE characteristic are known. We therefore suggest that the quantitative relationships between acoustic emission activity and fracture mechanics parameter, $K$ (stress intensity factor) and the fatigue parameter $\mathrm{JK}$ in fatigue are foundations of the new subject of quantitative acoustic emission-based life prediction methods. The macroscopic mechanisms of acoustic emission from fatigue are directly related to fracture mechanisms, as analyzed using fracture mechanics concepts. However, the mechanisms of damage must be known in some detail before such models can be applied to the prediction of fatigue life. That is, perhaps, the challenge for future research.

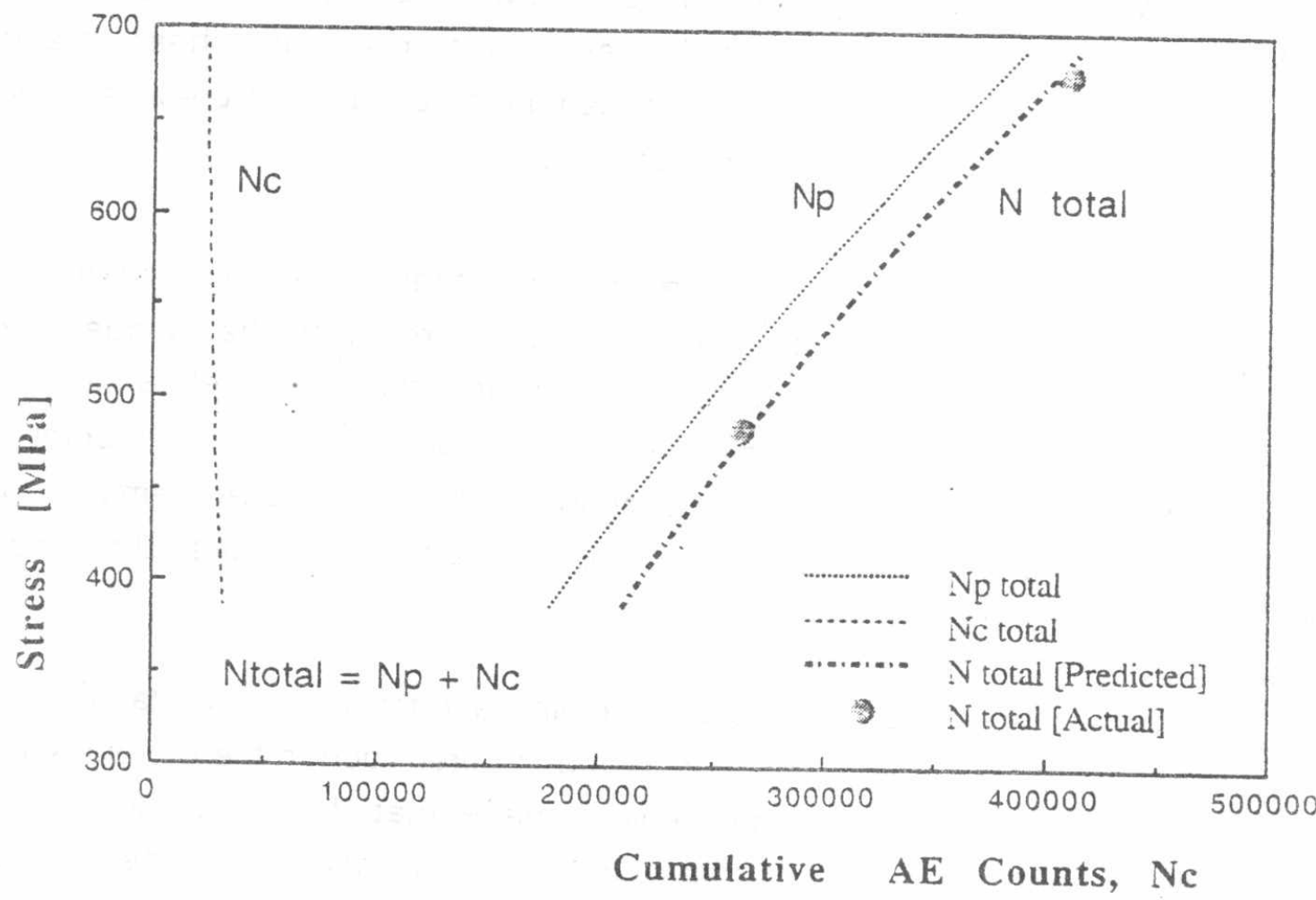

Figure 7: Comparison of Stress Versus the Predicted Number of AE Counts (Plastic Deformation Np, for Crack Extension Nc and The total Ntotal) and the Actual Data for As-Received Ti 15-3 Sample. 
The results of acoustic emission modeling suggests the importance of that powerful technique for damage monotoring and life prediction in the current aircractt applicationsns. Hidden and inaccessable damage that do not produce vissual indications can now be detected and easy to locate within a structural aerospece frame before, it grows and produces vissually observable indications, and detectable lengths without the need to disassembly. Other environmental effects will then be taken into consideration beside fatigue such as corrosion, corrosion fatigue, and more.

\section{CONCLUSIONS}

1. A fracture mechanics model has been developed for the prediction of the fatigue life of monolithic Ti-15-3 and $[0 / 90]_{2 S}$ Ti-15-3/SCS-6 composite. This model uses center crack and edge crack idealizations to simplify the fatigue crack growth and crack bridging analysis. Good agreement is obtained between the measured and predicted initiation and propagation lives in the monolithic Ti-15-3 matrix. Good agreement is also obtained between the predicted and the experimental composite fatigue lives when the interfacial shear stress is between $200 \mathrm{MPa}$ and $250 \mathrm{MPa}$, i.e. within the range of the experimentally determined interfacial shear strength. Generally good agreement is observed between the actual and the predicted fatigue lives when the effect of fiber bridging are assessed.

2- A fracture mechanics-based $A E$ model has been developed for fatigue life prediction. Acoustic emission counts are related directly to the fatigue driving force, $\mathrm{KK}$, in the model. The measured acoustic emission parameters are also related directly to fatigue fracture mechanisms. Theoretical and experimental data obtained for different stress ranges are shown to be in good agreement. This suggests that the acoustic emission can be used as a tool for life prediction and the estimation of a structure or component.

3- The results of acoustic emission modeling suggests the importance of that powerful technique for damage monotoring and life prediction in the current aircractt applications. Hidden and inaccessable damage that do not produce vissual indications can now be detected and easy to locate within structural aerospece frame before it grows and produces vissually observable indications, and detectable lengths without the need to disassembly. Other environmental effects will now be taken into consideration beside fatigue such as corrosion, corrosion fatigue, and 
others. This technique of damage history and life prediction will lead to a new maintenance philosphy under real service conditions. Characterization as well as location of fatigue damage under real service conditions will allow the airframe to utilize condition-based maintenance instead of programmed-depot maintence.

\section{REFERENCES}

1. Rabeeh, B. M.,"Acoustic Emission Study of Damage in aTi-15-3/SiC Composite and its Constituents", Ph. D. Thesis, OSU, June 1996.

2. Kantzos, P., Telesman, J. and Ghosn, L. Fatigue Crack Growth in a Unidirectional SCS-6/Ti-15-3 Composite, in Composite Materials: Fatigue and Fracture, Vol. 3, ASTM STP 1110, T. K. O'Brien, Editor, American Society for Testing and Materials, Philadelphia, 1991, pp.711-731.

3. The Stress Analysis of Crack handbook by Hiroshi Toda, Paris, P. C. and Irwin, G. R., 1985 Edition.

4. McMeeking, R. M. and Evans, A. G., Mech. Mater. 9, 217 (1990).

5. Yang, J. M, and Jeng, S. M., J. Metals, November 1989, Vol. 13, pp. 56-59.

6. Yang, C. J., Jeng, S. M., and Yang, J. M., Scripta Metall., 1990, Vol. 24, pp. $469-474$.

7. Saff, C. R., Harmon, D. M., and Johnson, W. W., J. Metals, 1988, Vol. 40, pp.58-63.

8. Walls, D., Bao, G. and Zok, F., "Effect of Fiber Failure on Fatigue Cracking in a Ti-SiC Composite," Scripta Metall., 1991, Vol. 25, p. 911

9. Soboyejo, W. O.,Venkateswara Rao, K. T., Sastry, S. M. L., and Richie, R. O., Metall. Trans. A, 24 (1993) pp. 585-600.

10. Hutchinson, J.W. and Jensen, H. M., " Models of Fiber Debonding and Pullout in Brittle Composites With Friction," Mechanics of Materials, Vol. 9, pp. 139-163 (1990).

11. Venkateswara Rao, K. T.,Soboyejo, W. O. , and Richie, R. O. , Metall. Trans. A, 23 (1992) pp. 2249-2257.

12. Majumdar, B. S., Newaz, G. M., and Ellis, J. R., Metall. Trans. A. 1993, Vol. 24 , pp. 1597-1610.

13. Pickard, S. M., Miracle, D. B., Majumdar, B., Kendig, K., Rothenflue, L. and Coker, D., "An Experimental Study of Residual Fiber Strains in Ti-15-3 Continuous Fiber Composites," Acta Metall. Mater., 1994.

14. Chan, K. S. . "Effect of Interface Degradation on Fiber Bridging of Composite Fatigue Cracks," Acta Metall. Mater. Vol. 41, No. 3, pp. 761-768,(1993).

15. Hamil, F., Bailon J. P., and Bassim, M. N., "Acoustic Emission mechanisms during High Cycle Fatigue." Engineering Fracture Mechanics. Vol.14(1981): pp 853. 15. Morton, H. L., Harrington R. M., and Bjeletich, J. G., "Acoustic Emission of Fatigue crack Growth." Engineering Fracture mechanics. Vol. 5, No. 3 (1973): pp. 691.

16. Sinclair, A. C. E., Connors, D. C, and Formby, C. F., Materials Science and Engineering. Vol. 28 (1977).

17. Lindley, T. C., Palmer, I. G. and Rivhards, C. E., Materials Science and Engineering. Vol. 32 (1978):pp 1. 